\title{
Erupted complex composite odontome: a rare case
}

\author{
Dr.Nitin S. Solanki ${ }^{1}$, Dr. Vipin Thakkar ${ }^{2}$, Dr. Deepak R. Kolte ${ }^{3}$ \\ ${ }_{1,2,3}$ (Dept of Oral \& Maxillofacial Surgery, Bharati Vidyapeeth Deemed University Dental College and Hospital, \\ Navi Mumbai, India)
}

\begin{abstract}
Odontomas are the most common types of Odontogenic tumors. Their prevalence exceeds that of all other Odontogenic tumors combined. Odontomas represent a hamartomatous malformation; the term refers to tumors of Odontogenic origin ${ }^{[1],{ }^{2]}}$. They are usually asymptomatic and are diagnosed on routine radiological examination as an incidental finding ${ }^{[1],[3]}$. Odontomas are further subdivided into compound and complex types depending on the extent of morpho differentiation or on the resemblance to normal teeth ${ }^{[4]}$. The compound odontome is composed of multiple small tooth like structures, the complex odontome consist of a conglomerate mass of enamel and dentine, which bears no anatomical resemblance to the tooth ${ }^{[3]}$. The eruption and infection of odontoma are uncommon, only few cases of erupted odontome are reported in the literature. We report a rare unusual case of an infected complex odontoma with concomitant eruption of odontoma in the oral cavity involving perforation of the mucosa with an impacted third molar which makes the presentation unusual. We also highlighted the significance of proper diagnosis as most of these rare tumors go unnoticed with incorrect treatment to the patient. The dentist should be familiar with common and unusual appearances of odontomas for proper diagnosis and management.
\end{abstract}

Keywords: (Benign, impacted tooth, Odontogenic tumor, Odontome, Tooth like structure)

\section{Case report:}

A 28 year old male patient reported to the Department of Oral \& Maxillofacial Surgery, with a chief complaint of pain, swelling and pus discharge in lower left back region in his oral cavity since one \& half years. The patient had no relevant medical history. The extra- oral examination revealed no significant findings, but intraorally it presented as a swelling in the left mandibular angle area, obliterating the part of the buccal vestibule. The intraoral presentation was a breach in the corresponding mucosa, through which the pus was extruded with expansion of the buccal and lingual cortex. Along with this clinically there was missing third molar on that side and the adjacent second molar was periodontally compromised. The unusual intraoral protrusion of the odontome from the third molar socket area was initially misdiagnosed as impacted third molar by a private practitioner, \& this misdiagnosis was likely due to a false clinical presentation of tooth like appearance of the odontome.

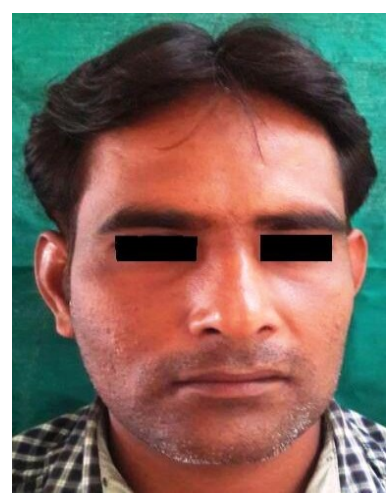

Fig.1 Preoperative photo

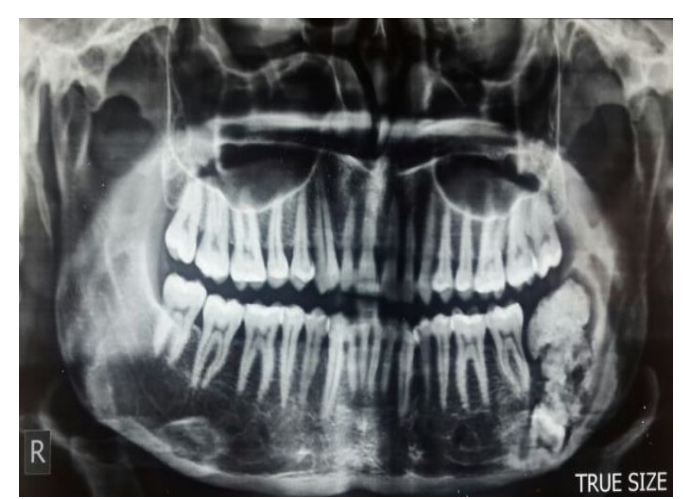

Fig.2 Preoperative OPG

A panoramic radiograph showed a uniform dense rounded radio-opacity measuring appx $4 \times 2.5 \mathrm{~cm}$ (Fig. 2) and on 3D CBCT the actual measurements were $20 \mathrm{~mm} \times 29 \mathrm{~mm} \times 19 \mathrm{~mm}$ (Antero- posterior x Superoinferior $x$ Bucco-lingual) associated with impacted 38. A 3D CBCT also showed well defined lobulated radioopaque mass seen in left mandible extending antero -posteriorly from distal of 37 to mid ramus and supero inferiorly from alveolar crest to the lower border of the mandible. The radiopaque mass was irregular in shape and surrounded by radiolucent capsule except on superior surface where there was no bony covering (mass exposed to oral cavity) (Fig. 3\&4). 


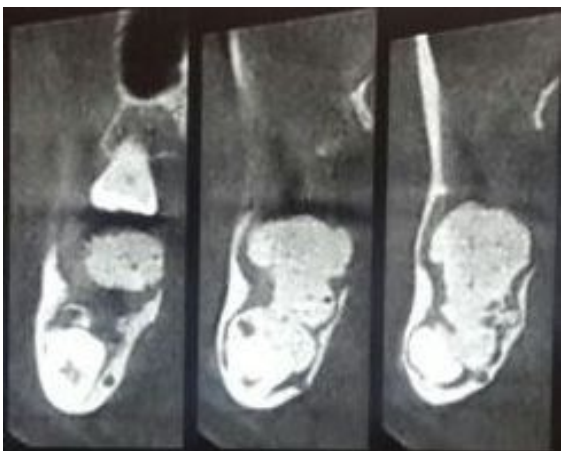

Fig. 3 Saggital view

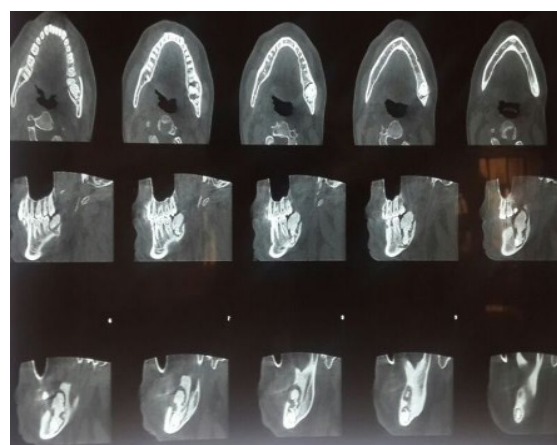

Fig. 4 Axial \& Saggital view

The mass was homogeneously radiopaque with small radiolucencies in between. There was expansion and thinning of buccal and lingual cortex. The mass had displaced 38 buccally and inferiorly to the lower border of the mandible and there was perforation of buccal cortex in that region (Fig. 3). The inferior alveolar nerve canal is displaced lingually and inferiorly with disruption in cortex at certain places in the area of the lesion. Also the neurovascular bundle was in very close proximity to the impacted third molar. There was condensation of bone surrounding the radioopque mass.

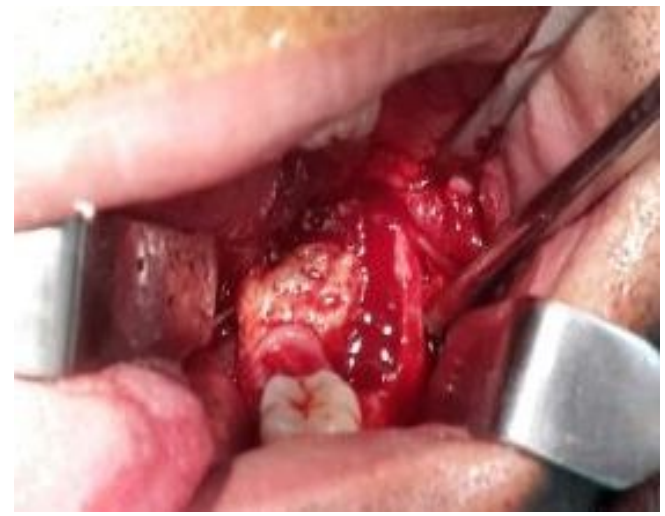

Fig. 5 Intraoperative photograph

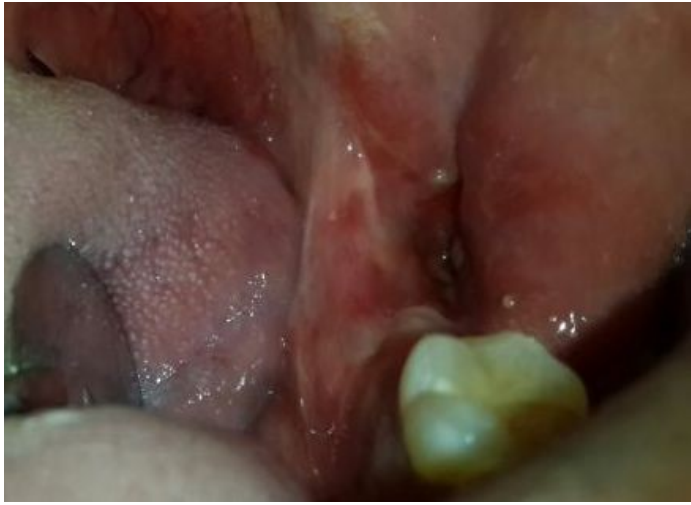

Fig. 7 Postoperatively after 6 months

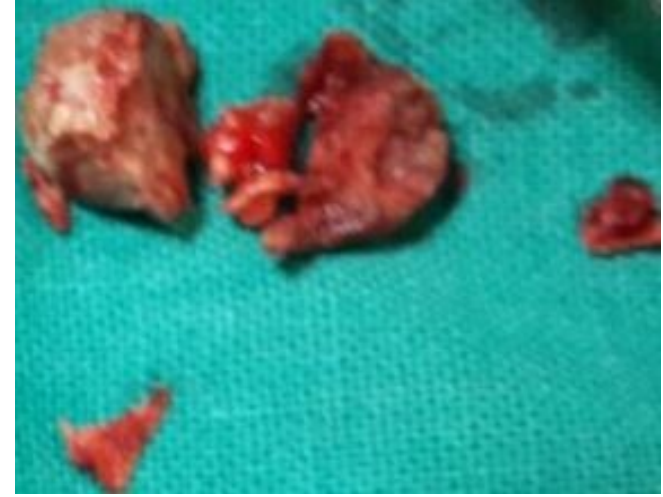

Fig. 6 Odontome mass removed in pieces

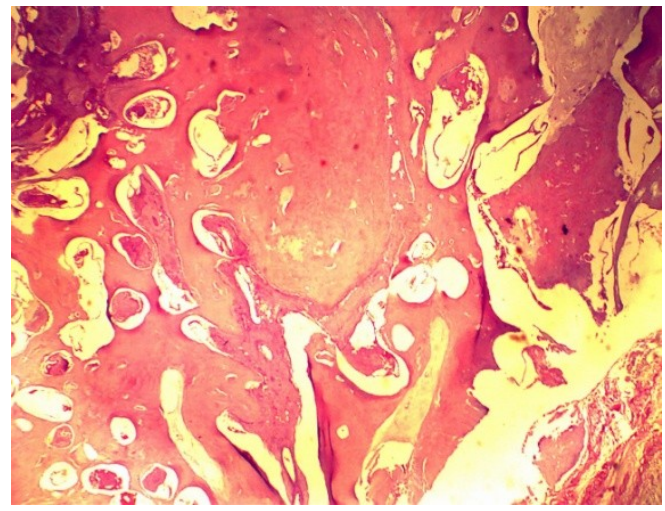

Fig. 8 Histopathologic picture 


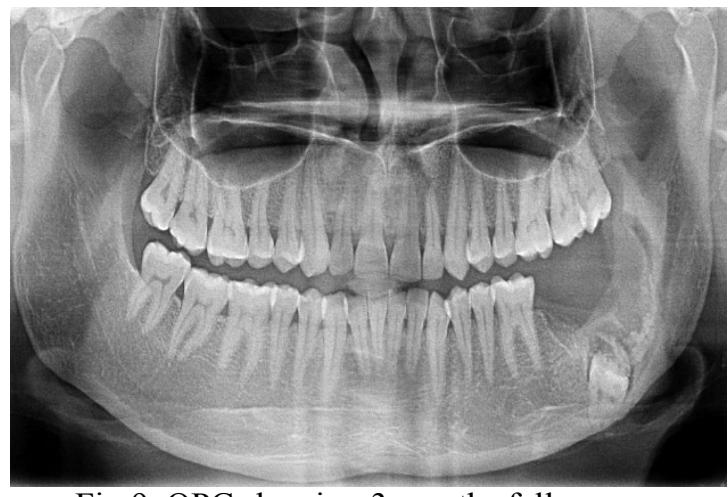

Fig.9. OPG showing 3 months follow-up

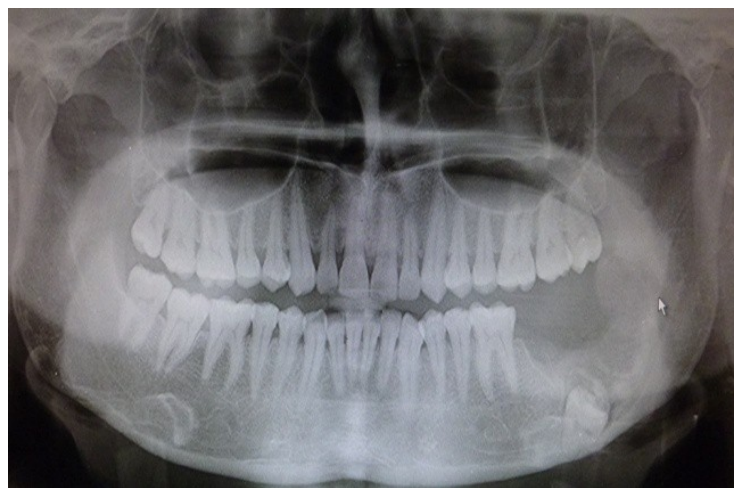

Fig. 10. OPG showing 6 months followup

\section{Discussion}

The WHO classification defines Complex Odontome as follows: "a malformation in which all the dental tissues are represented, individual tissues being mainly well formed but occurring in a more or less disorderly pattern" ${ }^{[5]}$. The etiology of Complex Odontomas is unknown. Several theories have been proposed, including local trauma, infection, family history, and genetic mutation (Gardeners's and Harmann's syndrome) ${ }^{[6]}$. It has also been suggested that odontomas are inherited from a mutant gene or interference, possibly postnatal with the genetic control of tooth development ${ }^{[7]}$. The relative frequency of Complex Odontome among odontogenic tumors varies between $5 \%$ and $30 \%$ which means that this lesion is one of the most commonest odontogenic lesion/malformation. The majority of cases $(83.9 \%)$ occur before the age of 30 with a peak in the second decade of life. The male: female ratio varies between 1.5: 1 and 1.6: 1. It is of interest to note that the majority of odontomas in anterior segment of jaw are compound composite, whereas the majority in posterior segment is Complex Odontome ${ }^{[8]}$. Interestingly, both type of odontomas occurred more frequently on the right side of jaw than on the left, such presentation was not seen in our case. Clinically Complex Odontome is a painless, slow-growing, and expanding lesion that is usually discovered on routine radiographs of the jaw bones, or the failed eruption of a permanent tooth which may lead to the diagnosis of this lesion. The first case of an erupted odontoma was described in 1980 by Rumel et al. The mechanism of odontome eruption appears to be different from tooth eruption because of the lack of periodontal ligament and root in odontoma. Therefore the force required to move the odontoma is not linked to the contractility of the fibroblasts, as in the case for teeth. Although there is no root formation in odontoma, its increasing size may lead to the sequestration of the overlying bone and hence occlusal movement or eruption. An increase in the size of the odontoma over time produces a force sufficient to cause bone resorption ${ }^{[2],[8]}$. Another reason for odontoma eruption could be the bone remodeling of the jaws. However, for this to occur dental follicle is required although indirectly, as it provides both the conductance and chemoattraction for the osteoclasts necessary for tooth eruption ${ }^{[9]}$. Radiologically, the compound odontoma appears as a collection of tooth-like structures while composite type appears as a calcified mass with a radiodensity similar to tooth structure; both are further surrounded by a narrow radiolucent zone ${ }^{[8]}$. However there was absence of any corticated border in our case as normally published in previous literature. Unerupted teeth are more commonly associated with compound composite odontoma. However, in our case, presence of unerupted tooth with Complex Odontome was seen. The lesion appears as a more or less amorphous, solitary mass of calcified material ${ }^{[4]}$. In some cases, the lesion shows a radiating structure. Rarely odontomas may form peripheral or soft tissue lesions in which they arise outside alveolar bone and may exfoliate or erupt. Such a rare erupted odontome was present in our case.

Microscopically, this lesion consists primarily of a well delineated, roughly spherical mass of a haphazard conglomerate of mature hard dental tissues. Some examples may include better-ordered, tooth-like structures. Clear spaces and clefts that probably contain mature enamel lost in the process of decalcification are often seen. In some sections at the periphery of the mass, islands of pulp tissue in association with cords and buds of odontogenic epithelium can be found. However, the usual high degree of differentiation of the dental tissues reflects the late stage of morpho differentiation and maturation of odontogenesis. A thin, fibrous capsule and, in some cases, a cyst wall is seen surrounding the lesion. In our case histopathologically we noticed excessive dentine - like tissue with dentinal tubules encircling of soft tissue resembling pulp. Also basophilic masses of cementum like tissues were seen in the adjoining fibrocellular connective tissue along with enamel space. Since the dentinal tissues were haphazardly arranged, overall picture was suggestive of Complex Odontome (Fig. 8).

Conservative surgical enucleation is considered to be the treatment of choice in most cases of Complex Odontome. During the removal of pathological structures in the mandibular retromolar region in an early age, care should be taken to preserve bone structures on the anterior border of the mandibular ramus, because it 
might impair jaw development. If needed, a control X-ray should be taken during the surgery. The prognosis is always good since these tumors do not tend to recur. As odontomas are often associated with impacted teeth, the possibility that eruption of the impacted tooth after a presumed obstructive odontoma has been surgically removed is an important issue. In our case, using an intraoral approach, a gingival mucoperiosteal flap was reflected and an occlusal- buccal window created to expose the mass (Fig. 5). The adjacent second molar was extracted as it was periodontally compromised. On exposure the mass appeared as a solitary, tooth- like structure. Ideally the mass needed to be removed in toto, but in our case owing to the proximity to the neurovascular bundle and in order to prevent the fracture of the jaw it was removed in sections (Fig. 6). Also third molar was spared due to following reasons.

1. To prevent damage to inferior alveolar neurovascular bundle $\&$ its function.

2. $3^{\text {rd }}$ molar being very close to inferior border, there were high chances of pathological fracture.

3. Allow eruption of 38 with possible orthodontic traction of 38 at the place of 37 .

Presently patient is under routine follow-up \& observation for eruption of third molar and shows adequate healing and no signs of infection or recurrence (Fig. 9\&10).

\section{Conclusion:}

An infected erupting complex odontoma in the mandibular third molar region may get misdiagnosed during clinical examination. This case being unusual because odontomas rarely erupt into the oral cavity \& less frequently on the left side of the mandible associated with impacted third molar. The conservative surgical removal of complex odontomas continues to be the treatment of choice. In the case of odontomas associated with impacted teeth, the latter should be preserved in wait of spontaneous eruption, followed by orthodontic traction ${ }^{8]}$

\section{References}

[1]. Agrawal B, Gharote H, Nair P, Shrivastav S. Infected complex odontome an unusual presentation. BMJ CASE REP: AUG 24; 2012.

[2]. Gokkulakrishnan, Sanjay Singh, Manpreet Singh \& Kumar Tathagat Singh. A rare case of odontome in 65yrs old lady. Nati J Maxillofac Surg. 2010 Jan- Jun; 1(1): 67- 70.

[3]. Hadas lehman, Joshua lustmann, Eran Regev. Removal of an extensive mandibular Odontoma using an intraoral approach. Quintessence International, Oral Surgery, Vol 44, no. 6, june 2013.

[4]. Audrey M. D'cruz, Shushmini Hegde, \& Urvashi A. Shetty. Large complex Odontoma. Sultan Qaboos Univ. Med. Jou, 2013, May; 13(2): E342-E345.

[5]. Vibha Singh, Satish Dhasmana, Shahabad Mohammad \& Nimisha Singh. The Odontomes: Report of 5 cases. Natl J Maxillofac Surg. 2010, Jul- Dec; 1(2): 157-160.

[6]. Monica Yadav, Pournima Godge, SM Meghana \& Sandip R Kulkarni. Compound Odontoma. Contemp Clin Dent. 2012 April; 3(suppl 1): S13-S15

[7]. Mengji Ashwin Kumar, Elluri Venkatesh, Dakshinamurty Shrikant, P Prashanth. Case Report of an infected erupting Complex Odontome \& its Evaluation Using CBCT. Universal Research Journal of Dentistry. Year: 2013, Vol. 3, Iss 3, pg: 134 - 137.

[8]. Dua Nisha, kapila Rishabh, Trivedi Ashwarya, Mahajan Sukriti, S.D Gupta. An unusual case of erupted Composite Complex Odontoma. Jounal of dental sciences and research, Vol 2, Iss 2, pg 1- 5.

[9]. Manoj vengal. Honey Arora, Sujoy Ghosh, Large Erupting Complex Odontoma: A case report. Jadc, March 2007, Vol. 73, N² 